\title{
Cutaneous larva migrans
}

INSERM

\section{Source}

INSERM. (1999). Orphanet: an online rare disease and orphan drug data base. Cutaneous larva migrans. ORPHA:423717

Cutaneous larva migrans is a rare parasitic disease characterized by sing le or multiple, linear or serping inous, erythematous, slightly elevated cutaneous tracks caused by the larval migration of various nematode species. Tracks are variable in length, generally a few millimeters wide and are frequently located on the feet (although any area of the body is possible). Patients typically present with severe, intractable pruritus, which, in some cases, may cause impaired concentration, loss of sleep, and mood disturbances. 